\title{
Ecología urbana de Triatoma infestans en Argentina. Asociación entre Triatoma infestans y palomares
}

\author{
Triatoma infestans urban ecology in Argentina. Association between Triatoma \\ infestans and pigeon cotes
}

\author{
Silvana L. Vallvé, Héctor Rojo, Cristina Wisnivesky-Colli
}

\begin{abstract}
Consejo Nacional de Investigaciones Cientificas y Técnicas (CONICET), Instituto y Museo de Ciencias Naturales, Facultad de Ciencias Exactas, Físicas y Naturales, Universidad Nacional de San Juan, Argentina (S.L.V.)

Municipalidad de la Capital de San Juan, Argentina (H.R.) CONICET, Unidad de Ecologia de Reservatorios y Vectores de Parásitos, Departamento de Ciencias Biologicas, Facultad de Ciencias Exactas y Naturales, Universidad Nacional de Buenos Aires (C.W.C.)
\end{abstract}

\begin{abstract}
Estudio se realizó en una área urbana de la Capital de la Provincia de San Juan, Argentina, en un barrio de 768 apartamentos distribuidos en edificios de 3 y 7 pisos que rodean una bodega central abandonada. Se capturaron 329 ejemplares de Triatoma infestans, 293 en 4 terrazas de torres de 7 pisos y 36 en el interior de la bodega, associados con numerosas palomas que colomizaban estos sitios. Los insectos se refugiaban en el guano acumulado entre bloques de cemento que cubren las terrazas y en la bodega dentro de toneles en desuso. Se identificaron dos focos principales de vectores associados con densos palomares: un 81,5\% del total de triatomas colectados se halló en una de las torres y un $11 \%$ en la bodega. A los 6 meses del tratamiento insecticida, resultaron positivas terrazas en las que previamente no se habia capturado $T$. infestans. El pertil alimentario de los triatomas muestra un predominio de ingestas simples sobre ave; en los edificios y en la bodega el $95 \%$ de los $T$. intestans analizados se alimentó sólo de ave; el resto fueron ingestas simples o dobles sobre humano, perro o gato. Ningún $T$. infestans presentó infección con Trypanosoma cruzi.
\end{abstract}

Triatoma. Ecologia de lectores. Palomas.

\section{Introducción}

La enfermedad de Chagas es una zoonosis caracteristica de las zonas rurales de América Latina. En general, se considera que en las ciudades las viviendas no están infestadas y existen solo focos ocasionales, generados a través de la introducción accidental de triatomas desde áreas rurales ${ }^{2}$. En zonas urbanas, la principal vía de transmisión del T.cruzi es la transfusión de sangre infectada?

Si bien esta caracterización epidemiológica describe adecuadamente muchas ciudades del continente y de nuestro país, no reviste por ello un carácter universal. Así, en la ciudad de San Juan, capital de la provincia homónima, ubicada en el Oeste de Argentina, el análisis de las solicitudes de rociado con insecticida dirigidas al Servicio Provincial de Control, durante los últimos años, indica que un alto porcentaje corresponde al Departamento Capital. Dichas solicitudes espontáneas se originan en la comprobación por parte de pobladores y/o funcionarios de la presencia de triatomas en sus viviendas o edificios públicos. El Departamento Capital, entre los años 1989 y 1992, acumuló un 25 a $36 \%$ del total de las notificaciones sobre la presencia triatomas registradas por el Servicio de Control. Debe tomarse en cuenta que casi la totalidad $(92,5 \%)$ de las 31.621 viviendas de la Capital. son construcciones urbanas. 
La ciudad de San Juan se caracteriza por la presencia de abundantes palomares en diversos locales y techos de los edifícios, aún en la zona céntrica.

En un trabajo previo realizado en un complejo de edificios de apartamentos de hasta 7 pisos de altura, un $51 \%$ de los habitantes encuestados manifestó que los adultos de $T$. infestans llegaban volando hasta su vivienda y el $64 \%$ de los pobladores censados capturó al menos una vez un T. infestans en su apartamento. La ocurrencia de fuertes vientos cálidos llamados "Zonda"s y la presencia de palomares en las terrazas son consideradas las principales causas de la aparición de estos triatomas en las viviendas.

Ante esta situación peculiar se decidió estudiar los mecanismos de infestación triatomínica en un conglomerado urbano, en el cual se registraron en los últimos 4 años numerosas notificaciones sobre la presencia de $T$. infestans. En dicha área se realizó un estudio de base preliminar al rociado insecticida y luego un seguimiento en el tiempo de las reinfestaciones.

\section{Material y Método}

\section{Área Estudiada}

El presente trabajo se realizó entre mayo y diciembre de 1993 en la ciudad Capital de la Provincia de San Juan, Argentina, situada al Oeste del país ( $31^{\circ} \mathrm{L}$.S. y $69^{\circ}$ L.O.). San Juan se halla en una de las zonas continentales más secas de Argentina, con un clima árido (tipo $\mathrm{BW}$ en la clasificación de Köeppen) y vegetación xerofítica típica de la Provincia Fitogeográfica de Monte. La precipitación pluvial media anual es de $96 \mathrm{~mm}$ con marcada estación invemal seca, la temperatura media anual de $18^{\circ} \mathrm{C}$ con una media de $26^{\circ} \mathrm{C}$ para enero. Existen grandes amplitudes térmicas diarias y estacionales, el verano es muy caluroso con valores máximos de hasta $45^{\circ} \mathrm{C}$.

En la zona es común la ocurrencia de vientos conocidos localmente como "Zonda", que se originan en el Océano Pacífico y se desplazan hacia la Cordillera de los Andes, cargados de humedad y con baja temperatura. Al ascender descargan su humedad en forma de tormentas y descienden aumentando su temperatura $\left(1^{\circ} \mathrm{C} / 100 \mathrm{~m}\right)$; llegan entonces, a las provincias limítrofes de Argentina, como fuertes vientos muy secos (menos del $5 \%$ de humedad) con elevadas temperaturas que alcanzan los $30^{\circ} \mathrm{C}$, en cualquier época del años.
El Departamento Capital de la provincia posee una superficie de $30 \mathrm{Km}^{2}$ y una población de 119.423 habitantes, distribuidos en 31.621 viviendas urbanas (Censo Nacional de Población, 1991).

La zona de estudio seleccionada fue el Barrio San Martin, ubicado hacia el Norte de la Ciudad. Durante el año 1989 el $7 \%$ de los pedidos de rociado del Departamento Capital al Servicio Provincial de Control provinieron de este barrio. Se trata de un complejo habitacional que abarca un área de 8 hectáreas integrado por 768 apartamentos distribuidos en 7 torres (T) de 7 pisos de altura, 6 intertorres y 6 sectores (S) de tres pisos, que circundan un gran espacio interior ocupado por una bodega abandonada (Fig. 1-a). En los techos e interior de la bodega y en las terrazas de algunos edificios existen grandes palomares.

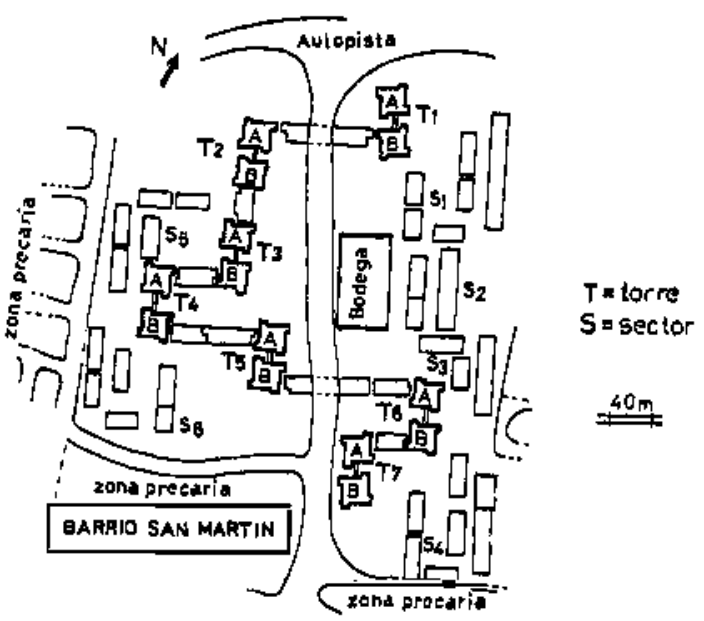

Figura 1a - Vista general de la distribución de los edificlos torre y la Bodega del Barrio San Martín.

Las torres están divididas en 2 monoblocks (A y B), unidos entre si por un puente cada dos pisos (Fig. 1-b). Un ascensor central comunica los dos monoblocks. Sus terrazas, que no tienen acceso al público, están cubiertas por bloquetas - medios blocks cortados longitudinalmente (Fig. 1-b), colocadas sobre una carpeta aislante de lana de vidrio y alquitrán. En cada terraza existen numerosas antenas de televisión que al agitarse con los vientos perforan la capa del aislante. Para repararla es necessario desplazar las bloquetas que en general no vuelven a ser colocadas en su lugar. Esto permite la instalación de numerosas palomas que anidan entre las bloquetas sueltas. 


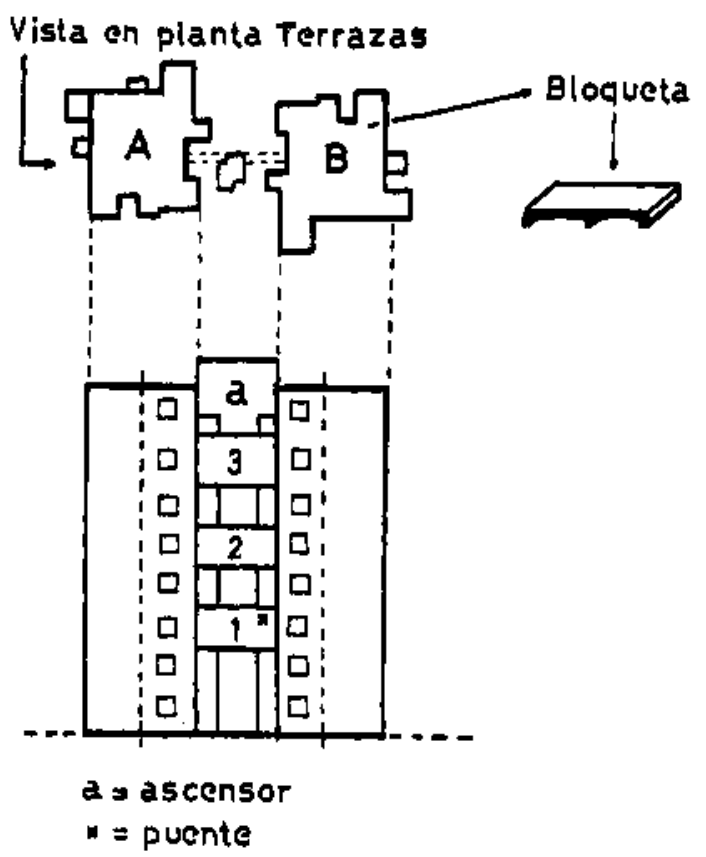

Flgura 1b - Detalle de una torre, sus terrazas y caracteristicas de las "bloquetas" bajo la que se refugian los $T$. infestans.

Desde hace 15 años, el Barrio San Martín está habitado por más de 2.600 personas $(2,2 \%$ de la población del Departamento Capital).

La bodega es un edificio que cubre un área de $2.800 \mathrm{~m}^{2}$, con paredes de ladrillo de 5 metros de alto y techo a dos aguas de chapas de zinc (Fig. 1c) con un sobretecho central elevado y abierto. En Jo alto de las paredes laterales hay 3 ventanas hacia el Oeste y 3 hacia el Este sin cerramientos. Posee un entrepiso al que se accede por una escalera de material. Tanto por las ventanas como por este techo ingresan y egresan palomas. En el interior de la bodega se encuentran seis toneles, repletos de papeles y escombros, que tiempo atrás servían para prensar el orujo de la uva. En estos toneles y en varios escondrijos y rincones del edificio anidan las palomas.

Todo el Barrio San Martín está rodeado por conglomerados de viviendas precarias situadas a distancias variables entre 2 y 150 metros (Fig 1-a).

\section{Muestreo Entomológico}

Tanto en las torres como en la bodega se realizó una primera inspección de los posibles focos de triatomas. En las terrazas se revisaron los nidos de palomas y los espacios entre las bloquetas y en la bodega los interiores de los toneles con palomares. Luego se realizó una evaluación ento- mológica (una hora-hombre) ${ }^{t 1}$ ya sea por disección total del hábitat o restringida a los lugares con nidos de aves.

En las terrazas se buscaron triatomas levantando las bloquetas en donde habia nidos de palomas, habitados o abandonados, examinando cuidadosamente el guano.

En la bodega, tres personas buscaron vectores simultáneamente durante 20 minutos sumando un total de 1 hora-hombre de evaluación.

Los insectos colectados en ambos sitios se guardaron en recipientes herméticos para realizar los estudios posteriores en el laboratorio. Luego fueron rociadas con deltametrina $-25 \mathrm{mg} / \mathrm{m}^{2}$. todas las zonas en las que se hallaron ejemplares de $T$. infestans. La semana siguiente se visitaron los iugares tratados para confirmar la efectividad del tratamiento insecticida y se colectaron los insectos muertos, para el análisis posterior de su perfil alimentario.

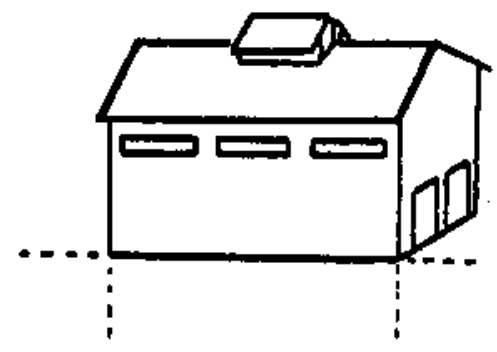

Flgura 1c - Bodega del Barrio San Martín.

En los apartamentos de los séptimos pisos de las terrazas infestadas se consultó sobre la tenencia de animales domésticos para relacionarlos con los resultados del perfil alimentario de los insectos colectados.

A los 6 meses del rociado se procedió a revisar todos los techos de los edificios y la bodega, siguiendo la misma metodología descripta, con el fin de detectar reinfestaciones y nuevos focos de triatomas.

\section{Trabajos de Laboratorio}

Todos los triatomas colectados se identificaron, contaron y clasificaron por estadio $y$ sexo. La presencia de $T$, cruzi fue determinada por observación microscópica de las heces de cada insecto $(400 \mathrm{x})^{10}$. Se obtuvo la sangre presente en el intestino anterior de cada triatoma, conservándola a $-22^{\circ} \mathrm{C}$, para la identificación de la fuente alimentaria por doble difusión en gel 
de agar, utilizando antisueros específicos ${ }^{10}$. Se analizaron los contenidos intestinales de cada triatoma contra 5 sueros de conejo antiproteínas sanguíneas de: humano, ave, perro, gato y roedor múrido.

\section{Resultados}

\section{Infestación por $T$. infestans en el Barrio}

Sobre un total de 7 torres del Barrio San Martín, se comprobó la presencia de T.infestans en 4 de ellas (1 A, 2A, 4B y 5A; Fig. 1-a) (Tabla 1). Se capituraron 293 ejemplares de $T$, infestans con un predominio de adultos $(64,2 \%)$ y de ninfas (N) de quinto estadío (N5) $(64,8 \%$ del total de ninfas). La relación de sexos fue 1,6 machos por cada hembra. Un $91,5 \%$ (268/293) del total de triatomas colectados se hallaron en la Torre $4 \mathrm{~B}$ y un $7,5 \%$ $(22 / 293)$ en la Torre $5 \mathrm{~A}$, orientada frente a la bodega a unos $40 \mathrm{~m}$. Los 3 adultos fueron encontrados en las torres 1 y 2 en nidos de palomas sobre el puente 3 (Fig. 1-b).

Tabla 1 - Triatoma infestans* capturados en los edificios torre del Barrio San Martín, Mayo de 1993 Departamento Capltal, San Juan, Argentina.

\begin{tabular}{|c|c|c|c|c|c|c|c|c|}
\hline \multirow[b]{2}{*}{ Sitio de colección } & \multicolumn{2}{|c|}{ Adultos } & \multicolumn{4}{|c|}{ Ninfas } & \multicolumn{2}{|c|}{ Total } \\
\hline & $\mathrm{H}$ & $M$ & 5 & 4 & 3 & 2 & Ad & $N$ \\
\hline $\begin{array}{l}\text { T 1A-puente } 3 \\
\text { T 2A-puente } 3 \\
\text { T 4B } \\
\text { T 5A }\end{array}$ & $\begin{array}{l}1 \\
60 \\
12\end{array}$ & $\begin{array}{r}2 \\
104 \\
9\end{array}$ & $\begin{array}{r}67 \\
1\end{array}$ & 15 & 5 & 17 & $\begin{array}{r}1 \\
2 \\
164 \\
21\end{array}$ & $\begin{array}{r}104 \\
1\end{array}$ \\
\hline \multirow[t]{2}{*}{$\begin{array}{l}\text { Totales } \\
(\%)\end{array}$} & $\begin{array}{c}73 \\
\text { (39) }\end{array}$ & $\begin{array}{l}115 \\
(61)\end{array}$ & 68 & 15 & 5 & 17 & $\begin{array}{l}188 \\
(64)\end{array}$ & $\begin{array}{l}105 \\
\text { (36) }\end{array}$ \\
\hline & & & & & & & 29 & 3 \\
\hline
\end{tabular}

* $\mathrm{H}=$ hembras, $\mathrm{M}=$ machos, $\mathrm{Ad}=$ adultos, $\mathrm{N}=$ ninfas $_{+}$ $T=$ torres

En la bodega se colectó un total de 36 adultos (21 hembras y 15 machos) (Tabla 2). Las mayores capturas, $83 \%$ del total, se realizaron en los toneles a y b que contenían numerosos trastos y nidos de palomas. Sin embargo, como muestra la tabla, también se localizaron adultos en los otros toneles que no ofrecían refugios tan abundantes.

En la evaluación entomológica de las terrazas una semana después del rociado sólo se capturaron 16 triatomas muertos. En la Torre 4, 10 adultos (5 hembras, 5 machos) y 4 ninfas ( $1 \mathrm{~N} 4,1 \mathrm{~N} 3$ y 2 $\mathrm{N} 2$ ); en la Torre 5, una hembra y una N2.
Tabla 2 - Triatoma infestans adultos capturados en la Bodega del Barrio San Martín, Junio de 1993, Departamento Capital, San Juan, Argentina.

\begin{tabular}{|c|c|c|c|c|c|}
\hline \multicolumn{2}{|c|}{ Lugar de captura } & \multicolumn{2}{|c|}{$\begin{array}{l}\mathrm{N}^{2} \text { de } T \text {. infestans } \\
\text { coleccionados }\end{array}$} & \multirow{2}{*}{\multicolumn{2}{|c|}{ Total* }} \\
\hline & & $\mathrm{H}$ & $M$ & & \\
\hline Tonel & $\begin{array}{l}a \\
b \\
c \\
d \\
e \\
f\end{array}$ & $\begin{array}{r}8 \\
10 \\
1\end{array}$ & $\begin{array}{l}5 \\
7 \\
1 \\
1 \\
1\end{array}$ & $\begin{array}{c}13 \\
17 \\
1 \\
1 \\
1 \\
3\end{array}$ & $(83,3 \%)$ \\
\hline Total & & 21 & 15 & 36 & \\
\hline
\end{tabular}

$\mathrm{H}=$ hembras; $\mathrm{M}=$ machos

* Una gran cantidad de ninfas capturadas no $8 \theta$ consignan en la tabla, ya que fueron incineradas debido a un error involuntario del encargado del edificio.

En la bodega, en la evaluación realizada 12 dias después del tratamiento, se encontró un total de 62 triatomas muertos, de los cuales el $94 \%$ eran adultos. Dos días después de esta evaluación, se colectaron 4 adultos vivos ( 3 machos y 1 hembra) en la escalera. Aunque es posible que estos insectos hubieran ingresado volando por las ventanas abiertas a 4 metros de altura, tampoco puede descartarse que por acción retardada del piretroide los triatomas escaparan de refugios propio de la bodega.

\section{Estudio de la Reinfestación a los 6 Meses del Tratamiento Insecticida}

En la nueva evaluación de las terrazas resultaron negativos todos los techos de 3 pisos de altura (sectores e intertorres), además de las terrazas de las Torres $1,2,4,5$ y 7 , que presentaban bloquetas ordenadas sin restos de nidos de palomas. En las Torres 4 y 5 , ya tratadas con deltametrina, no se encontraron triatomas. Sólo se colectaron 15 T. infestans en la Torre 3B, su puente $3 \mathrm{y}$ la Torre 6B que no habían sido rociadas previamente porque resultaron negativas en la primera evaluación. En la Torre 3B fueron capturadas 5 ninfas ( $2 \mathrm{~N} 3$ y $3 \mathrm{~N} 4)$ y en el puente 3 un macho. En la Torre 6B, al remover la totalidad de las bloquetas con guano muy fresco, se encontraron 7 adultos ( 2 hembras y 5 machos) y 2 ninfas ( $1 \mathrm{~N} 4$ y $1 \mathrm{~N} 5$ ) en dos focos en las esquinas orientadas hacia la bodega. Los adultos presentaban por observación externa escasa reserva alimentaria ${ }^{4,6}$.

\section{Perfil Alimentario de los Triatominos Capturados}

De los 156 ejemplares de $T$. infestans analizados ( $37 \%$ del total colectado), 90 fueron reactivos 
Teble 3 - Fuentes de alimentación identificadas para $T$, infestans según sitio de colección en el Barrio San Martin, 1993, Departamento Capital, San Juan, Argentina.

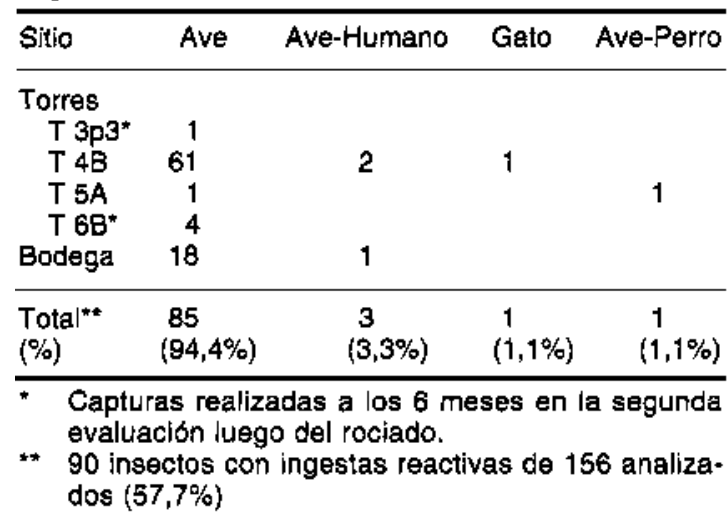

en la prueba de doble difusión (Tabla 3). Se observa un predominio de ingestas simples sobre un único hospedador, sólo $4 / 90$ ejemplares $(4,4 \%)$ presentaron comidas dobles. Tanto en los edificios como en la bodega se destaca el ave como principal fuente de alimentación, con un 94,4\% (85/90) de las ingestas analizadas.

En las consultas realizadas a los habitantes de los apartamentos inmediatamente inferiores a las terrazas cuyos triatomas dieron alimentación distinta de ave, resultó que en la Torre 4B criaban perros en 1 de 3 domicilios y en la Torre $6 \mathrm{~B}$ en 2 de 3 . En ninguna de las viviendas criaban gatos $u$ otro tipo de animal doméstico.

\section{Infección por Trypanosoma cruzi}

Todos los $T$. infestans capturados durante la evaluación de las torres y de la bodega del Barrio San Martín resultaron negativos a la infección por T. cruzi.

\section{Dlscusión y Conclusión}

Dentro del Barrio San Martín se identificaron dos focos principales de vectores: la bodega y la terraza de la Torre 4B. Ambos sitios poseŕan una población de palomas de alta densidad como fuente de alimentación disponible para los triatomas.

En el interior de la bodega las palomas encontraban un refugio seguro; se hallaba abandonada desde hacia más de 10 años y la perturbación externa era mínima, ya que sólo una persona entraba mensualmente a realizar una limpieza del guano acumulado. La presencia de numerosas aberturas permitia la entrada y salida constante de las aves. Este edificio mostraba una gran riqueza de escondites para los vectores, en sitios frescos y oscuros, con abundante comida en los nidos de palomas, lo que se verifica en los datos del perfil alimentario.

En la terraza de la Torre 4B la densidad de palomares era también alta y presentaba un elevado grado de desorden con numerosas bloquetas fuera de lugar, entre cuyos huecos las palomas podían construir nidos permanentes.

Las terrazas más infestadas, Torre 4B y Torre $5 \mathrm{~A}$, se hallaban próximas a las zonas con viviendas precarias circundantes, lo que sugiere que estas áreas podrían actuar como focos de dispersión de triatomas.

Se pudo establecer una clara vinculación entre el grado de desorden de las bloquetas, la colonización por parte de las palomas y la presencia de T. infestans. Así la terraza de la Torre 5A estaba más ordenada y presentaba menor número de nidos de palomas y de $T$. infestans que la Torre 4B. A su vez en la 5A se obșervó mayor número de bloquetas fuera de lugar que en el otro monoblock (5B), donde no se hallaron $T$. infestans.

La presencia mayoritaria de $T$. infestans adultos respecto a las ninfas ( 7 vs.2) en la Torre $6 \mathrm{~B}$, que no había estado colonizada por triatomas en el primer muestreo, sugiere que los insectos arribaron por vuelo a estas terrazas en búsqueda de fuentes de alimento, si bien no puede descartarse un transporte pasivo mediante las palomas. Refuerza esta hipótesis, el hecho de que los insectos presentaban escasa reserva alimentaria, es probable que estos llegaran ayunados desde alguna otra fuente de infestación densamente poblada, en coincidencia con estudios que afirman que la dispersión por vuelo se produce a densidades poblacionales altas cuando la disponibilidad de alimento es insuficiente?

En la bodega el predominio de alimentación simple sobre ave (18/19) indica que este sitio, ubicado en el centro del barrio, se comportaría como un foco estable de mantenimiento de triatominos a partir de una población permanente de palomas como fuente alimentaria. El único $T$. infestans que reacciono frente a los antisueros de hombre y ave podría haber llegado desde afuera, proveniente de casas o apartamentos periféricos.

El hecho de que la mayoría de los $T$. infestans asociados con palomas resultaran negativos a $T$. cruzi se explica por ser estas la fuente primordial de alimentación, pues tanto en las torres como en la Bodega el 95\% de las ingestas identificadas corresponden a ave. 
La asociación entre $T$. infestans y las aves de corral en zonas rurales es una hecho ampliamente observado. Las aves domésticas son una fuente importante de alimentación en distintas áreas geográficas ${ }^{12}$. Asimismo se ha probado una asociación significativa entre la presencia de $T$. infestans y la existencia de gallinas, tanto en Santa Cruz de la Sierra, Bolivia', como en Santiago del Estero, Argentina ${ }^{3}$.

En el presente trabajo se demuestra por primera vez una fuerte coexistencia entre palomas y $T$. infestans en un área urbana. La presencia de estos pájaros es tolerada en muchas ciudades de área endémica. Sin embargo, la directa asociación entre poblaciones de palomas y aparición de $T$. infestans en las ciudad de San Juan sugiere que es necesario realizar un seguimiento y control de estas aves y de los triatomas asociados en todos los barrios, aún en edificios de propiedad horizontal.

\section{Agradecimientos}

A Maria Mercedes Quel, Carmen Castro y Fabio Vásquez, en la revisión del manuscrito y con la colaboración de los revisores Miguel Guerra, Carlos Atencio y Roberto Guevara, de la Municipalidad de la Capital de San Juan.

\section{Referenclas Blbliográflcas}

1. DE MUYNCK, A.; RIBERA, B.; ZUNA, H.; VILLARROEL, G.; LAGRAVA, M.; LAGRAVA, M.S. de SERRANO, R.; VARGAS, J.; MELGAR, B.; NAVIA, J. Estudio epidemiológico de la enfermedad de Chagas en la Provincia de Vallegrande, (Departamento de Santa Cruz). In: Romero Davalos, A. ed. Enfermedad de Chagas. Cochabamba, Los Amigos del Libro, 1977. p. 115-50.

2. DIAS, J.C.P. Mecanismos de trasmisão en Trypanosoma cruzt. In: Brenner, Z. \& Andrade, $Z$. ed. Doença de Chagas. Rio de Janeiro, Guanabara Koogan, 1979. p. 152-74.

3. GAJATE, P.P.; BOTTAZZI, M.V.; PIETROKOVSKY, S.M.; WISNTVESKY-COLLI, C. Relação entre Triatoma infestans, aves domésticas e o homem num povoado de Santiago del Estero, Argentina. Cad. Saude Püblica, 10 (1): 53-7, 1994.

4. MONTENEGRO, S.S.C. de. Determinación de las reservas alimenticias en Triatoma infestans (klug, 1834) (Hemiptera: Reduviidae) en base a caractéres externos. I. Adultos. PHISIS (Buenos Aires) Sec. C., 41 (101): 15967, 1983

5. RETA, A.J. Descripción del agro-clima de San Juan. San Juan, Instituto Nacional de Tecnología Agropecuaria, Servicio de Agrometeorología, 1979.

6. ROSSEL, O.R. A comparision of the feeding performances of Rhodnitus protixus STAL and $R$. robustus LARROUSE (Hem.Triat.). s.l.p., 1984.
7. SCHMUNIS, G.A. Chagas disease and blood trasnfusion. In: Dodd, R. \& Baker, L,F, ed. Infection, immunity and blood tranfusion. New York, Alan R. Liss, 1985.

8. SCHWEIGMANN, N.; VALLVE, S.; MUSCIO, O.; GHILINI, M.; ALBERTI, A.; WISNTVESKYCOLII, C. Dispersal flight by Triatoma infestans in an arid area of Argentina. Med. Vet. Entomol, 2: 401-4, 1988.

9. SCHOFIELD, C.J. \& MATTHEWS, J.N.S Theoretical approach to active dispersal and colonization of houses by Triatoma infestans. J. Trop. Med. Hyg., 88: 211-22, 1985.

10. WISNIVESKY-COLLI, C ; GÜRTLER, R.E.; SOLARZ, N.; SAIOMON, D; RLIZ, A.M Feeding patterns of Triatoma infestans (Hemiptera: Reduviidae) in relation to transmission of American Trypanosomiasis in Argentina. J.Med. Entomol., 19: 645-54, 1982.

11. WISNIVESKY-COLLI, M.C.; PAULONE, I.; PEREZ, A.; CHUIT, R.; GLALTIERI, J.; SOLARZ, N.; SMITH, A.; SEGURA, E.I. A new tool for continous detection of the presence of triatomine bugs, vectors of Chagas disease. in rural households. Medicina (Buenos Aires), 47: 45-50, 1987 a.

12. WIS.NIVESKY-COLLI, C.; RUIZ, A.M.; LEDESMA, O.; GÜRTLER, R.E.; LAURICELLA, M. SALOMON, D.; SOLARZ, Nं; SEGURA, E.L. Eología doméstica de la tripanosomiasis americana: perfil alimentario de Triatoma infestans en un área rural de la Provincia de Santiago del Estero, Argentina. Rev. Soc. Bras. Med. Trop., 20: 31-9, 1987 b. 


\begin{abstract}
A study was undertaken in an urban area of the capital city of the province of San Juan, Argentina, in a housing complex of 768 flats distributed in bulldings of 3 and 7 floors each surrouding an abandoned central winery. A total of 329 Triatoma intestans were captured, 293 on 4 terraces of seven-floor-towers and 36 inside the winery, associated with the great number of pigeons which nest in those places. The bugs were sheltered in the dung accumulated between the cement blocks used to floor the terraces and inside the unused tuns in the winery. Two main bug foci were identified associated with the dense pigeon colonies: $81,5 \%$ of the $\mathrm{T}$, infestans collected were found in one of the towers (4B) and $11 \%$ in the central winery. After six months of insecticidal sprayind of the infested areas, those terraces in wich $\mathrm{T}$. intestans had not been previously found resulted gave positive results. The feeding profile of triatomines shows a predominance of simple bird blood meals; in the buildings and the winery $95 \%$ of the T. Infestans analized were ldentified for bird blood meals; the rest had fed on one or more sources: human, dogs or cats. None of the $\mathrm{T}$. infestans was infected by T. cruzi.
\end{abstract}

Triatoma, Ecology, vectors. Pigeons.

\title{
Resumo
}

Estudo realizado em local urbano da capital da Provincia de San Juan, Argentina, num bairro de 768 apartamentos distribuidos em prédios de 3 e 7 andares que cingem uma adega vinicola abandonada. Foram coletados 329 exemplares de Triatoma infestans, 293 em 4 terraços das torres de 7 andares e 36 no interior da adega, associados às numerosas pombas que colonizavam esses sitios. Os insetos refugiavam-se no excremento cumulado entre os blocos de cimento que cobrem os terracos $e$, na adega, dentro de tonéis fora de uso. Foram identificados dois focos principais de vetores, associados com densos pombais: $81,5 \%$ do total de triatomas coletados foram apanhados em uma das torres e $11 \%$ na adega. Apos 6 meses de tratamento com inseticida, foram positivos os terracos onde anteriormente nåo se tinha coletado o T. infestans. O perfil alimentar dos triatomas mostra predominio de sangue de aves; nos ediffcios e na adega $95 \%$ dos T. infestans analisados alimentaram-se somente de aves; os demais fizeram repastos simples ou mistos am humanos, cāes ou gatos. Nenhum T. Infestans apresentou infechâa com Trypanosoma cruzi.

Triatoma. Ecologia de Vetores. Pombos. 